\title{
The Canadian Partnership for Tomorrow Project: a pan-Canadian platform for research on chronic disease prevention
}

Trevor J.B. Dummer PhD, Philip Awadalla PhD, Catherine Boileau PhD, Camille Craig MSc, Isabel Fortier PhD, Vivek Goel MD, Jason M.T. Hicks MSc, Sébastien Jacquemont MD, Bartha Maria Knoppers PhD, Nhu Le PhD, Treena McDonald MSc, John McLaughlin PhD, Anne-Marie Mes-Masson PhD, Anne-Monique Nuyt MD, Lyle J. Palmer PhD, Louise Parker PhD, Mark Purdue PhD, Paula J. Robson PhD, John J. Spinelli PhD, David Thompson MSc, Jennifer Vena PhD, Ma'n Zawati LLM; with the CPTP Regional Cohort Consortium*

Cite as: CMAJ 2018 June 11;190:E710-7. doi: 10.1503/cmaj.170292

\begin{abstract}
BACKGROUND: Understanding the complex interaction of risk factors that increase the likelihood of developing common diseases is challenging. The Canadian Partnership for Tomorrow Project (CPTP) is a prospective cohort study created as a population-health research platform for assessing the effect of genetics, behaviour, family health history and environment (among other factors) on chronic diseases.
\end{abstract}

METHODS: Volunteer participants were recruited from the general Canadian population for a confederation of 5 regional cohorts. Participants were enrolled in the study and core information obtained using 2 approaches: attendance at a study assessment centre for all study measures (questionnaire, venous blood sample and physical measurements) or completion of the core questionnaire (online or paper), with later collection of other study measures where possible. Physical measurements included height, weight, percentage body fat and blood pressure. Participants consented to passive follow-up through linkage with administrative health databases and active follow-up through recontact. All participant data across the 5 regional cohorts were harmonized.

RESULTS: A total of 307017 participants aged 30-74 from 8 provinces were recruited. More than half provided a venous blood sample and/or other biological sample, and 33\% completed physical measurements. A total of 709 harmonized variables were created; almost $25 \%$ are available for all participants and $60 \%$ for at least 220000 participants.

INTERPRETATION: Primary recruitment for the CPTP is complete, and data and biosamples are available to Canadian and international researchers through a dataaccess process. The CPTP will support research into how modifiable risk factors, genetics and the environment interact to affect the development of cancer and other chronic diseases, ultimately contributing evidence to reduce the global burden of chronic disease. hronic disease prevention and individualized disease management are central to public health in the 21st century. ${ }^{1,2}$

However, the multifactorial etiology of most chronic diseases demands that we increase our understanding about how biology, genetics, environment and behaviours interact to affect disease risks and outcomes. Prospective cohort studies that track individuals over decades are important tools for exploring these complex interactions. ${ }^{3}$ One such tool is the Canadian Partnership for Tomorrow Project (CPTP) - a pan-Canadian prospective cohort that was envisioned in 2008 as a "population laboratory" 4,5 to support Canadian and international population health research in evaluating the genetic, behavioural and environmental causes of cancer and other chronic diseases.
The CPTP set out with an ambitious goal to recruit 300000 participants from 8 Canadian provinces, ${ }^{4}$ and to obtain a venous blood sample for biobanking from as many participants as possible. It is the largest prospective cohort ever created in Canada, and baseline data are now available to Canadian and international researchers. The aim of this article is to provide a baseline cohort profile of the CPTP, summarizing key sociodemographic, behavioural and health-related characteristics of the participants. We summarize the CPTP design and participant recruitment, the harmonization of the core data, the biorepository, and the procedures established to support data sharing with researchers. 


\section{Methods}

\section{Study design}

The CPTP study design has been described elsewhere. ${ }^{4}$ The primary inclusion criteria were that participants must be aged $30-74$ years at recruitment (initially the age range was 35-69 yr), a resident in 1 of the 8 collaborating provinces (Nova Scotia, New Brunswick, Prince Edward Island, Newfoundland and Labrador, Quebec, Ontario, Alberta and British Columbia) and able to complete questionnaires in English or French. The project was, from the outset, a confederation of 5 regional studies that leveraged existing and new investment to create a research platform based on a model similar to that used for the European Prospective Investigation into Cancer and Nutrition (EPIC) cohort. ${ }^{6}$ It brought together 2 existing cohorts (Alberta's Tomorrow Project ${ }^{7}$ and CARTaGENE $^{8}$ ), 2 cohorts that had begun pilot development (Ontario Health Study and BC Generations Project) and 1 newly created cohort (Atlantic Partnership for Tomorrow's Health [PATH] Study).

Following confirmation of funding in 2008, the study protocol, the core questionnaire and the standard operating procedures for obtaining physical measurements and biological specimens were developed. All participating regions began recruiting using the CPTP proto$\mathrm{col}$ in 2009. A range of recruitment and enrolment activities were used across 2 major approaches to obtain data from participants (Table 1).

\section{Table 1: Participant recruitment and data capture across the 5 regions of the Canadian Partnership for Tomorrow Project}

\section{CPTP region (province)}

Atlantic PATH (Nova Scotia, New Brunswick, Prince Edward Island, and Newfoundland and Labrador)

CARTaGENE (Quebec)

Ontario Health Study (Ontario)

Alberta's Tomorrow Project (Alberta)

BC Generations Project (British Columbia)

\section{Participant recruitment methods}

- Invitation from provincial health insurance provider (Nova Scotia only)

- Advertising

- Community events

- Workplace events

- Incentive programs (i.e., Air Miles)

- Invitation from provincial health insurance provider

- Incentive programs (i.e., Air Miles, gift cards)

- Email invitations to employees at large organizations

- Invitation emails and letters (based on purchased and commercial mailing lists)

- Advertising

- Community events

- Friend and family referrals

- Advertising

- Community events

- Workplace events

- Incentive programs (i.e., Air Miles)

- Invitation letters (based on purchased and commercial mailing lists)

- Recontact of all original participants in Alberta's Tomorrow Project for consent to the CPTP protocol

- Mailed or emailed personal invitation letters

- Awareness raising using earned media

- Email invitations to employees at large organizations

- Friend and family referrals
Core CPTP information collected

- Core questionnaire

- Consent for follow-up

and recontact

- Consent for linkage to health databases

- Venous blood sample

- Core questionnaire

- Consent for follow-up and recontact

- Consent for linkage to health databases

- Venous blood sample

- Core questionnaire

- Consent for follow-up and recontact

- Consent for linkage to health databases

- Venous blood sample

- Core questionnaire

- Consent for follow-up and recontact

- Consent for linkage to health databases

- Venous blood sample

- Height (standing, sitting), weight, waist and hip circumference

- Percentage body fat

- Grip strength

- Blood pressure

- Resting heart rate

- Core questionnaire

- Consent for follow-up and recontact

- Consent for linkage to health databases

- Venous blood sample
- Height (standing, sitting), weight, waist and hip circumference

- Percentage body fat

- Grip strength

- Blood pressure

- Resting heart rate
Additional biosamples

- Saliva

- Urine

- Dried blood spots

- Toenail clippings

- Saliva

- Urine

- Dried blood spots

- Saliva

- Urine

- Saliva

- Urine

- Dried blood spots

- Saliva

- Urine

Note: CPTP = Canadian Partnership for Tomorrow Project, PATH = Partnership for Tomorrow's Health

*Physical measurements were obtained using the following instruments: Seca stadiometer to measure height and sitting height, Lufkin steel tape to measure waist and hip circumference, Tanita BC- 418 body composition analyzer to measure weight and percentage body fat, Jamar dynamometer or Baseline digital hydraulic hand dynamometer to measure grip strength, and Omron HEM907XL to measure blood pressure. 
The first approach was based on participants visiting a study assessment centre, where they completed the core questionnaire, and provided blood and urine samples and a range of physical measurements, including height (standing and sitting), weight, waist and hip circumference, blood pressure, grip strength and percentage body fat measured using bioimpedance (Box 1). The use of study assessment centres in fixed locations was determined to be resource intensive and financially unsustainable. A 2010 evaluation concluded that to ensure the recruitment target of 300000 participants would be met within the available budget, an additional "distributed" recruitment and enrolment approach was required.

The second approach to recruitment was established in 2011 and introduced a 2-stage process by which participants first completed the core questionnaire (either online or paper), with the collection of a baseline blood sample scheduled for a later date for those able to attend an assessment centre or bloodcollection facility. When possible, physical measurements were also captured at the time of blood draw. This second approach provided opportunities for populations in rural areas and those who were unable to attend study assessment centres (typically in urban locations) to participate.

\section{Core data and biosamples collected}

The CPTP questionnaire included sociodemographic information, health information, behavioural and environmental factors, and self-reported anthropometric information (i.e., height, weight, and waist and hip circumference) (Box 1). Residential address, including postal code, was collected for most participants, allowing for future linkage of participant information to geospatial and environmental data sets.

During attendance at an assessment centre, or bloodcollection facility, a phlebotomist drew a nonfasting blood sample via arm antecubital venipuncture, either at or close to the time of enrolment or during a blood-collection follow-up phase. Urine samples (spot), collected in a sterile cup without preservatives, were often collected at the same time as blood. All regions collected a limited number of saliva samples, either by participants spitting into a sterile cup or using Oragene DNA saliva kits from DNA Genotek. Three regions (Atlantic PATH, Alberta's Tomorrow Project and CARTaGENE) collected dried blood spot samples on Whatman FTA cards from some participants. Atlantic PATH also collected toenail clippings.

Biosamples available in the regional biorepositories include SST (serum separator tube):serum, EDTA (ethylenediaminetetraacetic acid):plasma, EDTA:red blood cells, PST (plasma separator tube):plasma, cryopreserved whole blood, urine and toenails (Atlantic PATH only). DNA will also be made available from collected blood and saliva samples.

In 2014, a DNA quality-assurance exercise was undertaken to evaluate the efficacy of DNA obtained from the CPTP. A random selection of 1152 DNA source samples (buffy coat, whole blood, Whatman FTA cards) collected by each region during the different enrolment phases was selected for DNA extraction and analysis. A single laboratory was selected to perform the extraction and analysis. DNA was extracted using the Qiagen FlexiGene DNA kit following manufacturer instructions. DNA concentration was determined using PicoGreen (Invitrogen) according to manufacturer instructions. A nested $\beta$-globin polymerase chain reaction (PCR) protocol was selected for assessing the DNA quality. ${ }^{9}$

\section{Data access}

Project participants provided consent to passive follow-up through linkage with administrative health databases (e.g., cancer registries, vital statistics and provincial health insurance databases), to active follow-up (e.g., additional questionnaires and biological sample collection to enrich the baseline data), and to receive invitations to participate in other health-related research studies. Central to the concept of the population laboratory, participants consented to the future sharing of their deidentified data and samples with health researchers to support studies advancing knowledge in the etiology and outcomes of cancer and chronic disease.

Box 1: Questionnaire data, physical measurements and biological samples collected at baseline

\section{Core questionnaire}

- Sociodemographic: Date of birth, sex, ethnicity, education level, employment status, occupation, income, marital status

- Family history: Family characteristics, family history of cancer and other major diseases

- Health status: Self-rated health status, history of cancer and other diseases, reproductive history, prescribed medications, health-seeking behaviour (i.e., routine medical checkups, dental checkups, cancer screening)

- Behavioural factors: Alcohol, smoking, physical activity (International Physical Activity Questionnaire - Short Form), sleep, diet (fruit and vegetable servings per day)

- Environmental factors: Current residential address, environmental tobacco smoke, sun exposure, current occupation and shift work

- Self-reported anthropometry: Height, weight, waist and hip circumference

\section{Physical measurements}

- Anthropometry: Standing and sitting height, weight, body fat (bioimpedance), waist and hip circumference

- Blood pressure and heart rate: Two measurements 1 minute apart

- Grip strength: Right and left hand grip strength

\section{Biological samples}

- Venous blood: All regions (aliquots: serum, plasma, red blood cells, white blood cells or whole blood) (150 611 samples)

- Venous blood: BC Generations Project, CARTaGENE, Ontario Health Study (aliquots: whole blood in DMSO or separated lymphocytes in DMSO/FBS) (27 571 samples)

- Saliva: All regions (19 037 samples)

- Urine: All regions (100 975 samples)

- Dried blood spots: Atlantic PATH, CARTaGENE, Alberta's Tomorrow Project (65 472 samples)

- Toenail clippings*: Atlantic PATH (30 418 samples)

Note: $\mathrm{DMSO}=$ dimethyl sulfoxide, $\mathrm{FBS}$ = fetal bovine serum, $\mathrm{PATH}=$ Partnership for Tomorrow's Health.

*Access to Atlantic PATH toenail clippings is through the Atlantic PATH Project. 
A centralized online system was instigated for the CPTP that allows researchers who want to obtain core questionnaire data or biosamples from 2 or more regions to benefit from a "one stop shop" process for access requests. Detailed policies and procedures for review of data and sample requests are available, and access applications are currently handled via an online data and sample access portal (https://portal.partnershipfortomorrow.ca).

\section{Statistical analysis}

To assess the representativeness and generalizability of the CPTP cohort, various sociodemographic variables were compared with data for the Canadian population, ${ }^{10}$ for a similar age range, using the standardized difference method. ${ }^{11}$ An absolute standardized difference of 0.1 or less was interpreted as indicating no difference between the CPTP cohort and the Canadian population. The prevalence of common chronic diseases in the CPTP was compared with nationally available data for the prevalence of these diseases in a comparable age range. ${ }^{12,13,14}$

Both Alberta's Tomorrow Project and CARTaGENE had begun recruitment before the formation of the CPTP. Furthermore, logistical constraints during the initial period of study design (2009) and the introduction of a revised recruitment approach (2010/11) meant a number of versions of the core questionnaires were administered at different periods. To ensure that use of the CPTP core data are seamless, a rigorous data harmonization process was undertaken, described in detail elsewhere (unpublished data, 2018). ${ }^{15}$

The scale of the CPTP cohort (in excess of 300000 participants) from a population of 35 million in Canada reflects the need for adequate statistical power to support research questions that address the multifactorial interactions that underlie the chronic diseases that the CPTP will address. Provincial age- and sex-specific incidence rates for a number of cancers and other chronic diseases were applied to provincial age- and sex-specific participant counts in the CPTP to estimate the number of incident cases of common chronic diseases occurring in the CPTP during follow-up, accounting for the expected number of deaths.

\section{Ethics approval}

In the Canadian legal system, issues related to participant consent, privacy, confidentiality and research ethics are within the provincial domain. To maintain the highest standards of privacy and confidentiality that were consistent with provincial legislation, the Tri-Council Policy Statement and best practices for cohort-based research platforms, an ethics, legal and social issues committee was established. This committee informed, among other mandates, the development of regional consent forms and national access processes, as well as the conformity of CPTP processes with Canada's Tri-Council Policy Statement. ${ }^{4}$

\section{Results}

To date, the CPTP has successfully recruited 307017 participants from 8 provinces, with venous blood samples obtained from 150611 (49.1\%) (Table 2). Additionally, 33.7\% of participants have had physical measures taken (i.e., height, weight and percentage body fat).

Harmonization of the core questionnaire data was completed in December 2015. The number of participants for whom data are available varies by harmonized variable, depending on the version of the questionnaire used. From the 709 harmonized variables created, $23.4 \%$ were constructed for all participants and $58.7 \%$ were generated for at least 220000 participants.

Estimates of incident cases of common chronic diseases, accounting for expected number of deaths, occurring in the CPTP cohort during follow-up are provided in Appendix 1 (available at www.cmaj.ca/lookup/suppl/doi:10.1503/cmaj.170292/-/DC1). These estimates suggest that the CPTP is adequately powered for many common and less common chronic diseases.

\section{Cohort profile}

Similar to most large population-based cohort studies, the establishment of the CPTP relied on volunteers. For some characteristics - namely educational attainment, low income, working status and specific ethnicities - the cohort is unbalanced compared with the general population (Figure 1). Overall, more women (61.6\%) than

Table 2: Distribution of participants in the Canadian Partnership for Tomorrow Project by parent regional cohort

\begin{tabular}{|lcccc|}
\hline & \multicolumn{4}{c}{ Participants, no. (\%) } \\
\cline { 2 - 5 } & Men & Women & Total & $\begin{array}{c}\text { Provided } \\
\text { venous blood } \\
\text { sample }\end{array}$ \\
\hline Atlantic PATH & $10185(30.4)$ & $23344(69.6)$ & 33529 & $23897(71.3)$ \\
\hline CARTaGENE & $18995(44.8)$ & $23438(55.2)$ & 42433 & $29874(70.4)$ \\
\hline Ontario Health Study & $66172(40.5)$ & $97291(59.5)$ & 163463 & $41085(25.1)$ \\
\hline $\begin{array}{l}\text { Alberta's Tomorrow } \\
\text { Project }\end{array}$ & $13433(34.6)$ & $25351(65.4)$ & 38784 & $29193(75.3)$ \\
\hline BC Generations Project & $9028(31.3)$ & $19780(68.7)$ & 28808 & $26562(92.2)$ \\
\hline CPTP total & $117813(38.4)$ & $189204(61.6)$ & 307017 & $150611(49.1)$ \\
\hline Note: CPTP = Canadian Partnership for Tomorrow Project, PATH = Partnership for Tomorrow's Health.
\end{tabular}


men (38.4\%) were recruited, although this varied slightly between the regions (Appendix 2, available at www.cmaj.ca/lookup/suppl/ doi:10.1503/cmaj.170292/-/DC1). There were proportionately more participants in the older age groups, although $26.1 \%$ of participants were younger than 45 years (Appendix 2). In comparison to the general Canadian adult population, the CPTP participants were more highly educated, with substantially greater numbers completing a graduate-level education and significantly fewer with less than a high school education; however, given the size of the CPTP, there is considerable variation in this sociodemographic variable (Figure 1,
Appendix 2). Only $6.3 \%$ of participants had incomes less than $\$ 25000$, significantly lower than the national percentage, although the proportions with higher incomes were similar to national statistics (Figure 1, Appendix 2). Participants were predominantly white, reflecting the Canadian population, but the proportion of Aboriginal/Indigenous and Asian participants was not representative (Figure 1), which may limit the impact of CPTP for specific population groups. Most participants (63.1\%) spoke English as a first language, except for Quebec participants, who predominantly spoke French. Significantly more CPTP participants were retired

\section{Sociodemographic variables}

27. Income $>\$ 150000$

26. Income \$75 000-\$149999

25. Income $\$ 45000-\$ 74999$

24. Income \$25000-\$44999

23. Income $<\$ 25000$

22. Retired

21. Unable to work

20. Unemployed

19. Employed

18. Widowed

17. Divorced or separated

16. Single, never married

15. Married or living with partner

14. Graduate degree

13. Bachelor's degree

12. Diploma or certificate

11. High school

10. Less than high school

9. White

8. Latin American/Hispanic

7. Asian

6. Black

5. Aboriginal/Indigenous

4. Born outside Canada

3 Born in Canada

2. Female

1. Male

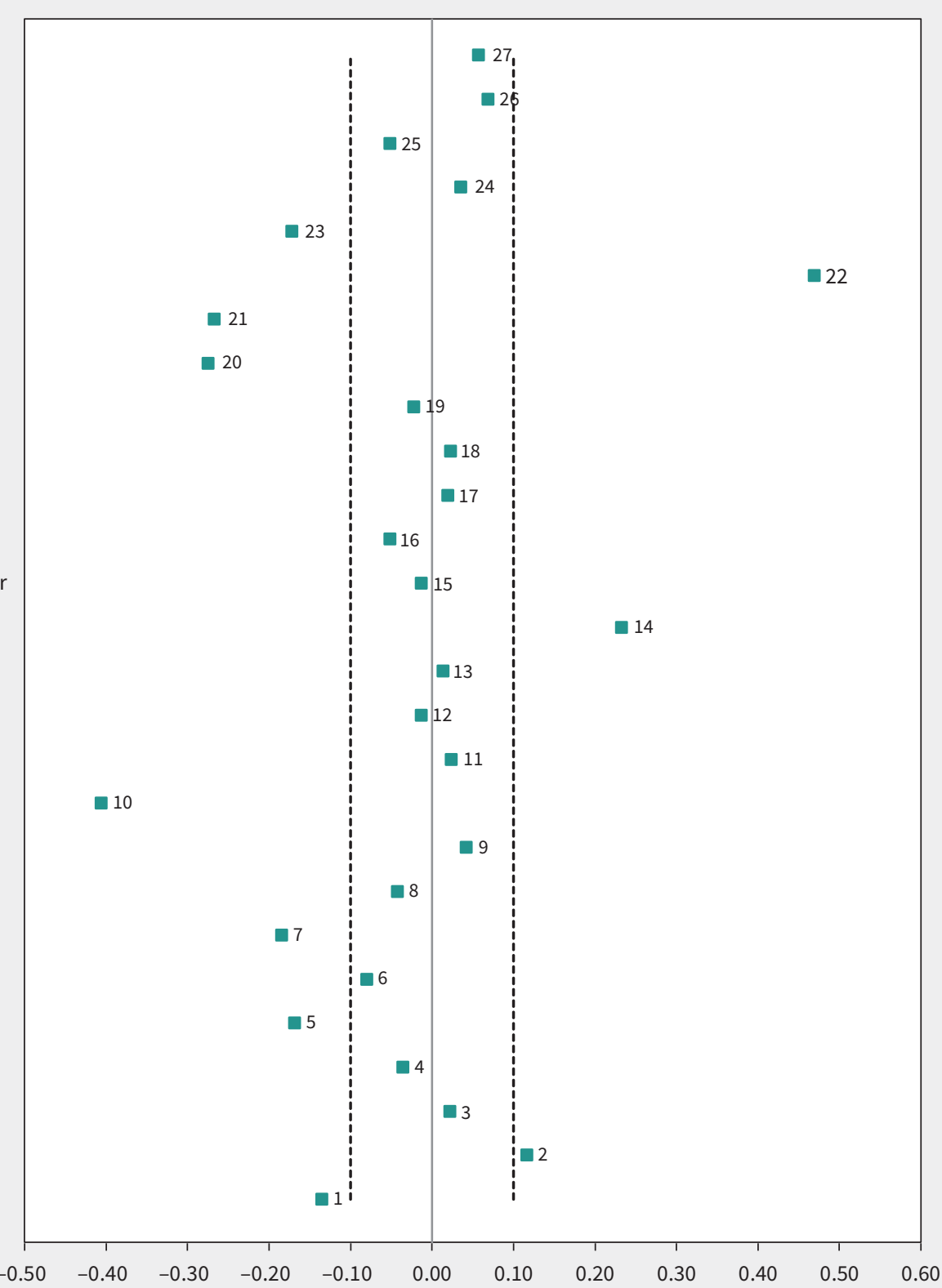

Figure 1: Standardized difference (CPTP and Canadian population) for specific sociodemographic variables. An absolute standardized difference greater than 0.1 is interpreted as indicating that there is a significant difference between the CPTP cohort and the Canadian population. For example, for the sociodemographic variable "graduate degree" (variable 14) there are significantly more individuals in the CPTP cohort who have a graduatelevel university degree compared to the general Canadian public. CPTP = Canadian Partnership for Tomorrow Project. 
compared with the general population, and fewer reported being unable to work or being unemployed (Figure 1).

There were minor variations in frequency distributions for a range of self-reported chronic diseases between the CPTP regions (Appendix 3, available at www.cmaj.ca/lookup/suppl/doi:10.1503/ cmaj.170292/-/DC1). Overall, proportions of participants reporting a diagnosis of a common chronic disease - for example diabetes (7.2\%), hypertension (22.6\%), arthritis (22.3\%) and asthma (12.1\%) - were comparable to Canadian estimates of these conditions (8.6\%, 23.3\%, 20.6\%, 7.9\%, respectively) in the similar age group of 45-64 years. There was greater regional variation in participants with a previous diagnosis of cancer. Overall, self-reported perceived quality of health reflects the national statistics for this age group, although there was some minor variation in perceived health status between regions (Appendix 3). For example, in Quebec considerably fewer participants rated their overall health as excellent or very good compared with elsewhere. Obesity was very prevalent; $36.4 \%$ of participants were overweight and $25.1 \%$ were obese, similar to national statistics for this age group (Appendix 3). However, there was considerable variation in overweight and obesity between regions, with the highest rates in Atlantic Canada and the lowest rates in $\mathrm{BC}$, consistent with the east-west obesity gradient reported in national surveillance data. There was a markedly lower proportion of current daily smokers in CPTP (9.6\%) compared with national data (19.2\%). A minority of CPTP participants (21.3\%) consumed 7 or more servings of fruit and vegetables per day, similar to national statistics for consuming 5 or more servings per day. Multimorbidity was common in the CPTP, with $26.2 \%$ reporting 2 or more chronic diseases. Clustering of 4 unhealthy lifestyle behaviours (low physical activity, current smoking, alcohol consumption almost daily, $\leq 3$ fruit/vegetable servings per day) was common, with $19.2 \%$ of participants reporting 2 or more unhealthy habits.

\section{DNA sample quality}

Quality and yield results of the 2014 DNA quality-assurance exercise for the biobank are presented in Appendix 4 (available at www. cmaj.ca/lookup/suppl/doi:10.1503/cmaj.170292/-/DC1). DNA was successfully extracted on all but 1 sample, with more than $98 \%$ having all 4 PCR products amplified, showing the high quality of the DNA obtainable and confirming its future utility for chip-based arrays and sequencing.

\section{Interpretation}

Primary recruitment for the CPTP is complete, and data and biosamples are available to Canadian and international researchers through a data-access process. The CPTP will support research into how modifiable risk factors, genetics and the environment interact to affect the development of cancer and other chronic diseases, ultimately contributing evidence to reduce the global burden of chronic disease.

Participant follow-up will ensure that the CPTP is a longitudinal cohort. As noted, follow-up is taking a number of forms, including both active (recontact for completion of additional questionnaires) and passive (linkage to provincial health insurance databases and disease registries to identify physician- diagnosed incident cases of disease). The first follow-up questionnaire was piloted in the summer of 2016 and administered 2016-2018. This included questions on health and behaviours that were asked in the core questionnaire to collect updated information that will support longitudinal analysis. It is anticipated that these data will be available in 2018.

Study participants have consented to linkage to their health data held in administrative health databases. All regions are actively engaged in working through the complex processes that will provide important information on cancer and other chronic disease incidence and health care use, and to allow this information to be made available for researchers. ${ }^{16}$ In all regions, access to some administrative health data linked to baseline questionnaires and biosamples is available via a regional cohort access request.

The CPTP has collected data and samples from populations across the diverse geography of Canada. The scope and scale of the CPTP provides a comprehensive chronic disease research platform that will enable both Canadian and international researchers to model health trajectories that take into account complex interactions between phenotypes, genotypes and environmental exposures. Noncommunicable chronic diseases result from the interaction of a multitude of factors, which on their own may have only modest effects. Large prospective populationbased cohorts provide a basis for exploring the complex multifactorial etiologies of chronic diseases. A cohort of more than 300000 , linked with administrative health databases, also provides scope for diseases surveillance across a geographically diverse population, which will support public health decisionmaking. There is also great potential for ancillary follow-up studies that use CPTP participants to test public health interventions and other health behaviour change initiatives.

The CPTP is a unique resource for Canada and other nations. It is characterized by a large sample drawn from across vastly differing regions of Canada - over half of whom have provided a blood sample or other DNA containing biosample - with physical measurements obtained from many. The CPTP is thus well positioned to support research to identify disease pathways and currently unknown interactions in these disease pathways. Such research is crucial in identifying targets for disease prevention and disease treatment. ${ }^{17}$ The CPTP is an important platform for the identification of biomarkers (using "omics" and other high throughput technologies $)^{18}$ for the early detection of disease, thereby supporting the development of the tailored disease prevention and therapeutic approaches envisaged under the precision medicine concept. ${ }^{19}$

The CPTP is similar in scope and scale to other national longitudinal population health cohorts, such as the well-established UK Biobank, ${ }^{20}$ which includes 500000 participants aged $40-69$ years, and the German National Cohort, ${ }^{21}$ which is currently in recruitment phase. Large population-based cohorts are essential to more fully understand the complex etiology of cancer and other chronic diseases. ${ }^{22,23}$

\section{Limitations}

The voluntary recruitment to the CPTP may affect the external validity of the study and limit generalizability, although the standardized data capture tools help ensure internal validity. A strength of the cohort study design is that all analytic comparisons 
are internal, which limits selection and information bias, ${ }^{24}$ and because of the large sample size there is heterogeneity in many important sociodemographic variables, which supports generalizability to larger populations.

While the voluntary nature of the CPTP has resulted in a more highly educated and slightly more affluent population compared with the general public, the prevalence of common chronic diseases and obesity - similar to national rates - and occurrence or multimorbidity and unhealthy habits indicates that the "healthy volunteer effect" 24 is unlikely to substantially influence long-term disease outcomes in CPTP, with the exception of smoking-related disease.

The greater regional variation observed in participants with a previous diagnosis of cancer likely reflects difference in participant recruitment and outreach, as opposed to regional variation in the prevalence of cancer; for instance, self-reported cancer rates were highest in British Columbia, which generally has the lowest rates of cancer in Canada. ${ }^{25} \mathrm{~A}$ common questionnaire was used across the CPTP, and hence it is unlikely that participants in different provinces would report cancer differently.

In common with the UK Biobank, ${ }^{26} \mathrm{EPIC}^{27}$ and other large population-based cohorts, ${ }^{28,29}$ it was not feasible to collect only fasting samples from population-based participants, many of whom would be more likely to come for blood draw in the late afternoon or evening. Therefore, nonfasting samples were collected and participants were asked when they last ate or drank; this enables identification of subsets that will be likely to meet researchers' criteria for specific assays. The decision to collect nonfasting blood samples does not diminish the value of the CPTP biobank, as these samples can be used for many different assays. For example, nonfasting measures of specific lipids (highdensity lipoprotein [HDL] cholesterol, total:HDL cholesterol ratio, apolipoprotein A-1 and triglycerides, have been shown to be highly effective predictors of cardiovascular disease risk. ${ }^{30}$ In relation to diabetes diagnosis, the measurement of hemoglobin $\left(\mathrm{HbA}_{1 \mathrm{c}}\right)$ is becoming standard practice and does not require fasting. ${ }^{31}$ Further, many inflammation-related biomarkers can be assessed in nonfasting blood samples. ${ }^{32}$

\section{Conclusion}

The CPTP has successfully created a large population health laboratory containing data for more than 300000 Canadians aged 30-74 years, with baseline data, longitudinal follow-up data, measurements and biosamples available to researchers via an access process. As the CPTP matures over the next several decades, some participants will develop cancer, heart disease and other chronic health conditions, some will die, and some will remain disease-free. Given adequate ongoing funding participants will provide additional information and biological samples, and disease-specific end points and health care use will be identified from linked administrative health databases. Future nested case-control studies will allow investigators to combine the health, environment and behavioural information and biological samples - collected at baseline and follow-up - to elucidate interactions between factors that cause chronic diseases. Importantly, the CPTP is a platform that is sufficiently large and com- prehensive to allow Canada to participate as an equal partner in the extremely large consortia that are needed to study geneenvironment, gene-gene and environment-environment interactions in the etiology of cancers and other chronic diseases.

\section{References}

1. Hamburg MA, Collins FS. The path to personalized medicine. N Engl J Med 2010; 363:301-4.

2. Hood L, Friend SH. Predictive, personalized, preventive, participatory (P4) cancer medicine. Nat Rev Clin Oncol 2011;8:184-7.

3. Manolio TA, Bailey-Wilson JE, Collins FS. Genes, environment and the value of prospective cohort studies. Nat Rev Genet 2006; 7:812-20.

4. Borugian MJ, Robson P, Fortier I, et al. The Canadian Partnership for Tomorrow Project: building a pan-Canadian research platform for disease prevention. CMAJ 2010;182:1197-201.

5. Potter JD. Epidemiology informing clinical practice: from bills of mortality to population laboratories. Nat Clin Pract Oncol 2005;2:625-34.

6. Bingham S, Riboli E. Diet and cancer - the European Prospective Investigation into Cancer and Nutrition. Nat Rev Cancer 2004;4:206-15.

7. Robson PJ, Solbak NM, Haig TR, et al. Design, methods and demographics from phase I of Alberta's Tomorrow Project cohort: a prospective cohort profile. CMAJ Open 2016;4:E515-27.

8. Awadalla P, Boileau C, Payette Y, et al. CARTaGENE Project. Cohort profile of the CARTaGENE study: Quebec's population-based biobank for public health and personalized genomics. Int J Epidemiol 2013;42:1285-99.

9. Greer CE, Peterson SL, Kiviat NB, et al. PCR amplification from paraffin-embedded tissues. Effects of fixative and fixation time. Am J Clin Pathol 1991;95:117-24.

10. National Household Survey Profile, 2011. Ottawa: Statistics Canada; 2013. Available: www5.statcan.gc.ca/olc-cel/olc?Objld=99-004-X2011001\&ObjType=46\& lang=en (accessed 2018 Mar. 1).

11. Austin PC. Using the standardized difference to compare the prevalence of a binary variable between two groups in observational research. Commun Stat Simul Comput 2009;38:1228-34

12. Table CANSIM 105-0501: Health indicator profile, annual estimates, by age group and sex, Canada, provinces, territories, health regions (2013 boundaries) and peer groups. Ottawa: Statistics Canada; 2016. Available: www5.statcan.gc.ca/cansim/ a26? lang=eng\&retrLang=eng\&id=1050501\&paSer=\&pattern=\&stByVal=1\&p1= $1 \&$ p2=37\&tabMode=dataTable\&csid= (accessed 2016 May 26).

13. Navaneelan T, Janz T. Adjusting the scales: obesity in the Canadian population after correcting for respondent bias. Ottawa: Statistics Canada; 2014. Available: www.statcan.gc.ca/pub/82-624-x/2014001/article/11922-eng.pdf (accessed 2015 Apr. 14).

14. Twells LK, Gregory DM, Reddigan J, et al. Current and predicted prevalence of obesity in Canada: a trend analysis. CMAJ Open 2014;2:E18-26.

15. Fortier I, Raina P, Van de Heuvel ER, et al. Maelstrom Research guidelines for rigorous retrospective data harmonization. Int J Epidemiol 2017;46:103-5.

16. Doiron D, Raina P, Fortier I. Linkage Between Cohorts and Health Care Utilization Data: Meeting of Canadian Stakeholders workshop participants. Linking Canadian population health data: maximizing the potential of cohort and administrative data. Can J Public Health 2013;104:e258-61.

17. Pearce MS, McConnell JC, Potter C, et al. Global LINE-1 DNA methylation is associated with blood glycaemic and lipid profiles. Int J Epidemiol 2012;41:210-7.

18. Horgan RP, Kenny LC. 'Omic' technologies: genomics, transcriptomics, proteomics and metabolomics. Obstet Gynaecol 2011;13:189-95. doi:10.1576/ toag.13.3.189.27672.

19. Collins FS, Varmus H. A new initiative on precision medicine. N Engl J Med 2015; 372:793-5.

20. Sudlow C, Gallacher J, Allen N, et al. UK Biobank: an open access resource for identifying the causes of a wide range of complex diseases of middle and old age. PLoS Med 2015;12:e1001779.

21. German National Cohort (GNC) Consortium. The German National Cohort: aims, study design and organization. Eur J Epidemiol 2014;29:371-82.

22. Burton PR, Hansell AL, Fortier I, et al. Size matters: just how big is BIG?: quantifying realistic sample size requirements for human genome epidemiology. Int J Epidemiol 2009;38:263-73.

23. Khoury MJ, Freedman AN, Gillanders EM, et al. Frontiers in cancer epidemiology: a challenge to the research community from the Epidemiology and Genomics Research Program at the National Cancer Institute. Cancer Epidemiol Biomarkers Prev 2012;21:999-1001. 
24. Lindsted KD, Fraser GE, Steinkohl M, et al. Healthy volunteer effect in a cohort study: temporal resolution in the Adventist Health Study. J Clin Epidemiol 1996;49:783-90.

25. Canadian Cancer Society's Advisory Committee on Cancer Statistics. Canadian Cancer Statistics 2015. Toronto: Canadian Cancer Society; 2015. Available: www.cancer.ca/ /media/cancer.ca/CW/cancer\%20information/cancer\%20101 /Canadian\%20cancer\%20statistics/Canadian-Cancer-Statistics-2015-EN.pdf ?la=en (accessed 2016 Feb. 9).

26. Elliott P, Peakman TC; UK Biobank. The UK Biobank sample handling and storage protocol for the collection, processing and archiving of human blood and urine. Int J Epidemiol 2008;37:234-44.

27. Crowe FL, Allen NE, Appleby PN, et al. Fatty acid composition of plasma phospholipids and risk of prostate cancer in a case-control analysis nested within the European Prospective Investigation into Cancer and Nutrition. Am J Clin Nutr 2008;88:1353-63.
28. Bradbury KE, Skeaff CM, Crowe FL, et al. Serum fatty acid reference ranges: percentiles from a New Zealand national nutrition survey. Nutrients 2011;3: 152-63.

29. Chen Z, Chen J, Collins R, et al.; China Kadoorie Biobank (CKB) collaborative group. China Kadoorie Biobank of 0.5 million people: survey methods, baseline characteristics and long-term follow-up. Int J Epidemiol 2011;40:1652-66.

30. Mora S, Rifai N, Buring JE, et al. Fasting compared with nonfasting lipids and apolipoproteins for predicting incident cardiovascular events. Circulation 2008;118:993-1001.

31. Saudek CD, Herman WH, Sacks DB, et al. A new look at screening and diagnosing diabetes mellitus. J Clin Endocrinol Metab 2008;93:2447-53.

32. Herder C, Baumert J, Zierer A, et al. Immunological and cardiometabolic risk factors in theprediction of type 2 diabetes and coronary events: MONICA/KORA Augsburg case-cohort study. PLoS One 2011;6:e19852.
Competing interests: Trevor Dummer reports grants from the Canadian Partnership Against Cancer during the conduct of the study. Sébastien Jacquemont reports grants from the Canadian Institutes of Health Research, grants from Genome Quebec, funding from the Cable Public Affairs Channel during the conduct of the study, and funding from Novartis and GW Pharmaceuticals outside the submitted work. Treena McDonald reports grants from Canadian Partnership Against Cancer during the conduct of the study. Paula Robson reports grants from the Alberta Cancer Foundation, grants from Government of Alberta via the Alberta Cancer Prevention Legacy Fund, grants from Alberta Innovates - Health Solutions via the Alberta Cancer Prevention Legacy Fund and grants from Canadian Partnership Against Cancer during the conduct of the study. Ma'n Zawati reports grants from Canadian Partnership Against Cancer during the conduct of the study.

This article has been peer reviewed.

Affiliations: School of Population and Public Health (Dummer), University of British Columbia, Vancouver, BC; Ontario Institute for Cancer Research (Awadalla); CARTaGENE (Boileau), Montréal, Que.; Research Institute of the McGill University Health Centre (Craig, Fortier); Research and Innovation, University of Toronto (Goel); Ontario Agency for Health Protection and Promotion (Goel); Atlantic PATH, Dalhousie University (Hicks), Halifax, NS; Centre hospitalier universitaire Sainte-Justine (Jacquemont); Centre of Genomics and Policy, McGill University (Knoppers, Zawati), Montréal, Que.; BC Cancer Research Centre (Le, McDonald), Vancouver, BC; Public Health Ontario (McLaughlin), Toronto, Ont.; Institut du cancer de Montréal, Université de Montréal (Mes-Masson); Pediatrics, CHU Sainte-Justine Research Center (Nuyt), Montréal, Que.; School of Public Health, University of Adelaide (Palmer), Adelaide, Australia; Department of Medicine, Dalhousie University (Parker); Division of Cancer Epidemiology and Genetics, National Cancer Institute (Purdue), Bethesda, Md.; CancerControl Alberta, Alberta Health Services (Robson, Vena), Edmonton, Alta.; Population Oncology, BC Cancer (Spinelli), Vancouver, BC; Atlantic PATH, Dalhousie University (Thompson), Halifax, NS
Contributors: All of the authors contributed to the manuscript conception and design. Trevor Dummer wrote the first draft and revised the manuscript. Camille Craig and Isabel Fortier provided data for Appendices 3 and 4 , and contributed to drafting the harmonization section. Treena McDonald and AnneMarie Mes-Masson provided data for Appendix 4 and contributed to drafting the biobank section. Bartha Knoppers and Ma'n Zawati contributed to drafting the access section. Catherine Boileau generated data for Appendix 1 . All of the authors were involved in reviewing drafts and suggesting revisions. All of the authors approved the final version to be published and agreed to be accountable for all aspects of the work.

*The CPTP Regional Cohort Consortium: Marilyn Borugian PhD, Heather Bryant MD, Christine M. Friedenreich PhD, Richard P. Gallagher MA, Heather K. Whelan MSc, Ilona Csizmadi PhD, Karen A. Kopciuk PhD, Nigel T. Brockton PhD, Craig Earle MD, Don Willison ScD, Heather Manson MD, Jack V. Tu MD, Jane Irvine DPhil, John Granton MD, Julia Knight PhD, Julian Little PhD, Lorraine Lipscombe MDCM, Karen Menard PhD, Kelly McDonald MA, Nancy Kreiger PhD, Sutapa Mukherjee MBBS, Tomas Paus MD PhD, William Hodge MD, Zdenka Pausova MD, Yves Payette MSc, Tharsiya Nagulesapillai BSc, Chenwei Gao MPH, Rikki Bharadwaj MSc, Sally Stasi BSc, Nina Chan BNSc, Melissa Moore OCAD

Funding: Funding for the Canadian Partnership for Tomorrow Project was from the Canadian Partnership Against Cancer, Health Canada, the Ontario Institute for Cancer Research, Alberta Health Services, the Alberta Cancer Prevention Legacy Fund, Alberta Cancer Foundation, Genome Quebec and the BC Cancer Foundation.

Data sharing: The CPTP data and biosamples are available to researchers through a data and sample access process.

Acknowledgements: The authors thank the participants in the Canadian Partnership for Tomorrow Project (CPTP) across the 5 regional cohorts who generously donated their time, information and biological samples. The CPTP is a success because of the participants' commitment. The authors also thank the many individuals who worked diligently to help build the CPTP, including the staff of the CPTP National Coordination Centre (Canadian Partnership Against Cancer); the Maelstrom Research Harmonization team and the Harmonization Committee; the Biosamples Standing Committee; the Ethics, Legal and Social Issues Committee; the CARTaGENE team, the Atlantic Partnership for Tomorrow's Health team, the Ontario Health Study team, the Alberta's Tomorrow Project team and the BC Generations team. Specifically, the authors thank the following: Melissa Ketler, Sanaz Vaseghi, Wendy Powell, Geraldine Lo Siou, Deep Monga, Will Rosner, Charlene Karvonen, Olga Maciejewski, S. Elizabeth McGregor, Ahmed El-Sohemy, Alan Bocking, Albert Wong, Andrea Gershon, Andriy Samokhvalov, Aristotle Voineskos, Art Slutsky, Barry Pakes, Bob Mann, Bob Reid, Brian Levine, Cam Mustard, Carole Madeley, Chi-Ming Chow, Chris Ardern, Claude Messier, Clodagh Ryan, Daniel Mueller, Dennis Cvitkovitch, Dianne Lougheed, Duncan Stewart, Fiona Miller, Gustavo Saposnik, Jaro Kotalik, Jennifer Kuk, Jennifer Olajos-Clow, Julia Spaniol, Karen Urbanoski, Kathy PichoraFuller, Katya Feder, Ken Chapman, Kwame McKenzie, Lisa Cicutto, Lisa Schwartz, Lisa Sweet, Mary Tierney, Matthew Stanbrook, Michael Szego, Natasha Rajah, Patricia Main, Patrick Davidson, Paul Demers, Paula Harvey, Pierre Cote, Ray Copes, Rayjean Hung, Robert Maunder, Robert Nolan, Rod Breau, Roger McIntyre, Ross Upshur, Sandra Black, Shelley Harris, Simone Vigod, Smita Pakhalé, Sonia Anand, Stanka Fitneva, Teresa To, Todd Norwood, Tony Hanley, Ubaka Ogbogu, Claude Laberge, Erika Kleiderman, Alison Caulfield, Celine Moore, Jacques Magnan, Tanya Flanagan and Donna Turner.

Disclaimer: Matthew Stanbrook, who is acknowledged by the authors, is a deputy editor for CMAJ and was not involved in the editorial decision-making process for this article.

Accepted: Apr. 12, 2018

Correspondence to: Trevor Dummer, trevor. dummer@ubc.ca 